University of Nebraska - Lincoln

DigitalCommons@University of Nebraska - Lincoln

Publications, Agencies and Staff of the U.S.

Department of Commerce

U.S. Department of Commerce

2011

Inbreeding effective population size and parentage analysis

without parents

Robin S. Waples

NOAA Fisheries, robin.waples@noaa.gov

Ryan K. Waples

Casa Azul

Follow this and additional works at: https://digitalcommons.unl.edu/usdeptcommercepub

Part of the Environmental Sciences Commons

Waples, Robin S. and Waples, Ryan K., "Inbreeding effective population size and parentage analysis without parents" (2011). Publications, Agencies and Staff of the U.S. Department of Commerce. 325. https://digitalcommons.unl.edu/usdeptcommercepub/325

This Article is brought to you for free and open access by the U.S. Department of Commerce at DigitalCommons@University of Nebraska - Lincoln. It has been accepted for inclusion in Publications, Agencies and Staff of the U.S. Department of Commerce by an authorized administrator of DigitalCommons@University of Nebraska - Lincoln. 


\title{
ANALYTICALAPPROACHES Inbreeding effective population size and parentage analysis without parents
}

\author{
ROBIN S. WAPLES* and RYAN K. WAPLES† \\ *NOAA Fisheries, Northwest Fisheries Science Center, 2725 Montlake Blvd. East, Seattle, WA 98112, USA, +Casa Azul, 10056 \\ Dibble Ave N, Seattle, WA 98177, USA
}

\begin{abstract}
An important use of genetic parentage analysis is the ability to directly calculate the number of offspring produced by each parent $\left(k_{i}\right)$ and hence effective population size, $N_{\mathrm{e}}$. But what if parental genotypes are not available? In theory, given enough markers, it should be possible to reconstruct parental genotypes based entirely on a sample of progeny, and if so the vector of parental $k_{i}$ values. However, this would provide information only about parents that actually contributed offspring to the sample. How would ignoring the 'null' parents (those that produced no offspring) affect an estimate of $N_{\mathrm{e}}$ ? The surprising answer is that null parents have no effect at all. We show that: (i) The standard formula for inbreeding $N_{\mathrm{e}}$ can be rewritten so that it is a function only of sample size and $\sum\left(k_{i}^{2}\right)$; it is not necessary to know the total number of parents $(N)$. This same relationship does not hold for variance $N_{\mathrm{e}}$. (ii) This novel formula provides an unbiased estimate of $N_{\mathrm{e}}$ even if only a subset of progeny is available, provided the parental contributions are accurately determined, in which case precision is also high compared to other single-sample estimators of $N_{\mathrm{e}}$ (iii) It is not necessary to actually reconstruct parental genotypes; from a matrix of pairwise relationships (as can be estimated by some current software programs), it is possible to construct the vector of $k_{i}$ values and estimate $N_{\mathrm{e}}$. The new method based on parentage analysis without parents (PwoP) can potentially be useful as a single-sample estimator of contemporary $N_{e}$ provided that either (i) relationships can be accurately determined, or (ii) $\sum\left(k_{i}^{2}\right)$ can be estimated directly.
\end{abstract}

Keywords: contemporary $N_{\mathrm{e}}$, relationship, reproductive success, sib reconstruction, single-sample estimator

Received 25 May 2010; revision received 12 August 2010; accepted 18 August 2010

\section{Introduction}

In the last two decades, parentage analyses made possible by highly polymorphic molecular markers have produced many novel insights (reviewed by Jones et al. 2010). In a typical parentage analysis, multilocus genotypes are scored in both progeny and potential parents, and these data are used to 'assign' progeny to parents, either through a probabilistic framework or by excluding other potential parents as impossible, given the rules of Mendelian inheritance. Parentage analysis can provide diverse types of information, including the direct (demographic) calculation of effective population size $\left(N_{\mathrm{e}}\right)$, which is one of the most important parameters in evolutionary biology but also one of the most difficult to estimate. For a monoecious population with random selfing, inbreeding effective size is given by (Crow \& Denniston 1988, Equation 1; Caballero 1994):

Correspondence: Robin Waples, Fax: (206) 860 3335;

E-mail: robin.waples@noaa.gov

$$
N_{\mathrm{e}}=\frac{\bar{k} N-1}{\bar{k}-1+V_{k} / \bar{k}}
$$

where $N$ is the number in the parental generation, and $\bar{k}$ and $V_{k}$ are the mean and variance of the number of offspring contributed by each parent $\left(k_{i}\right)$. If genetic methods can allow one to identify the number of offspring produced by each parent, the vector of $k_{i}$ values can be used to calculate $\bar{k}, V_{k}$ and $N_{\mathrm{e}}$ using eqn (1) (or slight variations that apply to other mating systems).

Now consider a variation to this scenario in which the parents cannot be sampled, but parents can be reconstructed from genotypes of the progeny-that is, a parentage analysis without parents (PwoP). It does not appear that a complete parentage analysis without parents has been conducted to date, but under some conditions, it is possible to reconstruct genotypes of some missing parents (e.g., Emery et al. 2001; Wang 2004). With enough highly variable markers, this can be quite feasible, provided that a sufficient number of known siblings are available (Jones \& Avise 1997; DeWoody et al. 2000) or at least one of the parents can be genotyped (Myers \& 
Zamudio 2004; Jones 2005; Tatarenkov et al. 2008; Eriksson et al. 2010). For the moment, assume that it is possible, from a sample of progeny alone, to reconstruct all genotypes from contributing parents, and from this, the vector of $k_{i}$ values for those parents. But there is an important difference between this vector and the vector of $k_{i}$ values in a standard parentage analysis: Because the PwoP vector only provides information about parents that actually produced progeny, it contains no elements with $k_{i}=0$, which represent null parents (those that produced no progeny that survived to the stage at which they were enumerated). In a typical population, the parental generation might have included an unknown (but potentially large) number of individuals that produced no surviving offspring. For example, in an ideal population (in which $V_{k} \approx \bar{k}=2$ ), on average, about $13 \%$ of parents will produce no offspring that survive to maturity, and the proportion should be higher in most natural populations (in which $V_{k}$ typically will be larger than $\bar{k}$ ). What effect do these missing parents have on calculations of $N_{\mathrm{e}}$ based on PwoP? The surprising answer is that they have no effect at all: it is not necessary to know anything about null parents to compute $N_{\mathrm{e}}$. We demonstrate this with a simple analytical proof, which results in a novel formula for inbreeding effective size that does not depend on N. Further, we use analytical and numerical methods to demonstrate the following:

1 Even an incomplete (but random) sample of progeny provides an unbiased estimate of inbreeding $N_{\mathrm{e}}$ using the novel formula;

2 The same insensitivity to null parents does not apply to variance effective size, which reflects the number in the progeny generation and hence varies with the number of progeny sampled;

3 It is not necessary to actually reconstruct parental genotypes to calculate $N_{\mathrm{e}}$ using PwoP; from a matrix of pairwise relationships (as can be estimated by current software programs), it is possible to construct the vector of $k_{i}$ values and estimate $N_{\mathrm{e}}$;

4 Assuming sibling relationships can be accurately determined, precision of the new method based on parentage analysis without parents appears to compare favourably with single-sample estimators of $N_{\mathrm{e}}$ currently in use. However, accurately determining relationship categories is a very challenging problem.

\section{Effective population size and null parents}

Assume a population has $N$ potential parents, and one wants to calculate effective population size using eqn (1). Further, assume that we have sampled the entire population of offspring and that the two parents of each offspring can be identified. It is then straightforward to construct the vector of $k_{i}$ values that describes the number of offspring produced by each parent. Parametric formulas for mean and variance of $k$ can be expressed as follows:

$$
\begin{gathered}
\bar{k}=\frac{\sum k_{i}}{N} \\
V_{k}=\frac{\sum\left(k_{i}^{2}\right)}{N}-\left[\frac{\sum k_{i}}{N}\right]^{2}
\end{gathered}
$$

Because we are interested in effective size of a particular population in a particular generation, we follow Crow \& Denniston (1988) and treat the $k_{i}$ as fixed, so in computing the population variance, the $N /(N-1)$ sample-size correction is not used. Substituting the above into eqn (1) leads to

$$
N_{\mathrm{e}}=\frac{\frac{\sum k_{i}}{N} N-1}{\frac{\sum k_{i}}{N}-1+\frac{\frac{\sum\left(k_{i}^{2}\right)}{N}-\left[\frac{\sum k_{i}}{N}\right]^{2}}{\frac{\sum k_{i}}{N}}}
$$

which, after simplifying, yields

$$
N_{\mathrm{e}}=\frac{\sum k_{i}-1}{\frac{\sum\left(k_{i}^{2}\right)}{\sum k_{i}}-1}
$$

As each diploid progeny has two parents, $\sum k_{i}=2 S$, where $S$ is the number of progeny, and eqn (2a) can also be written as

$$
N_{\mathrm{e}}=\frac{2 S-1}{\frac{\sum\left(k_{i}^{2}\right)}{2 S}-1}
$$

The surprising consequence of eqn (2) is that inbreeding $N_{\mathrm{e}}$ does not depend on parental population size $(N)$ - only on the two summation terms $\sum k_{i}$ and $\sum\left(k_{i}^{2}\right)$. Further, as $\sum k_{i}$ is determined by the sample size, the new formula shows that inbreeding $N_{\mathrm{e}}$ depends on a single unknown term $\left(\sum\left(k_{i}^{2}\right)\right.$ ). Because this term is not affected by any $k_{i}=0$, it follows that parents that contribute no offspring have no effect on inbreeding $N_{\mathrm{e}}$. This means that $N_{\mathrm{e}}$ can be computed directly from the progeny generation based only on information about parents that actually leave offspring, without knowing what parental $N$ was. The independence of inbreeding $N_{\mathrm{e}}$ with $N$ is a curious result, given that whether one includes null parents or not affects $N, \bar{k}$ and $V_{k}$. However, these parameters change in a correlated way such that the overall effect creates no change in $N_{\mathrm{e}}$. To illustrate, consider a diploid population with $N=10$ adults, for which the vector of $k_{i}$ values is [5, 1, 2, 4, 2, 0, 2, 1, 0,3]. For these data, $\bar{k}=2.0, V_{k}=2.40$ and $N_{\mathrm{e}}=8.64$ from eqn (1). Note that two of the parents produced no offspring. If we ignore 
those null parents, then $N=8, \bar{k}=2.5, V_{k}=1.75$-all different compared to the scenario that considered all 10 potential parents. However, the resulting $N_{\mathrm{e}}$ for the scenario that ignores null parents is 8.64 -identical to the value based on all the parents.

It is easy to show that this same approach does not work (at least in a general way) with variance $N_{\mathrm{e}}$. The analogue to eqn (1) for variance $N_{\mathrm{e}}$ in a monoecious population is (Crow \& Denniston 1988, Equation 19)

$$
N_{\mathrm{e}}=\frac{4 N^{\prime}-\bar{k}}{2\left[1+V_{k} / \bar{k}\right]}
$$

where $N^{\prime}$ is the number of progeny rather than the number of parents $(N)$. If we note that $N^{\prime}=N \bar{k} / 2$, this reduces to a form that is expressed in terms of the number of parents:

$$
N_{\mathrm{e}}=\frac{\bar{k}(2 N-1)}{2\left[1+V_{k} / \bar{k}\right]}
$$

Substituting the formulas for $\bar{k}$ and $V_{k}$ above does not lead to an expression that is independent of $N$, as occurs with inbreeding $N_{\mathrm{e}}$. Because variance $N_{\mathrm{e}}$ reflects the number in the progeny generation (Crow 1954; cf eqn 3), it is proportional to $\bar{k}$ and hence depends on the sample size of progeny. For this reason, calculations of variance $N_{\mathrm{e}}$ based on parent-offspring data are generally meaningful only if the mean and variance of $k$ are scaled to what they would be expected to be in a population of constant size (Crow \& Morton 1955). The effects on PwoP analyses can be seen by considering the hypothetical example described earlier. Using data for all 10 parents, $N_{\mathrm{e}}$ calculated from eqn (4) is the same as it is for inbreeding $N_{\mathrm{e}}$ (8.64), as should always be the case for random mating populations of constant size. However, when the two null parents are excluded, variance $N_{\mathrm{e}}$ rises to 11.03, reflecting the higher $\bar{k}$ for the eight parents that actually produced offspring. In contrast, the formula for inbreeding $N_{\mathrm{e}}$ (eqn 1 ) has an extra $\bar{k}$ term in denominator that automatically adjusts for the effects of changes in population size. As a consequence, calculation of inbreeding $N_{\mathrm{e}}$ from large samples of progeny does not require one to first rescale $\bar{k}$ and $V_{k}$ (Waples 2002), and $\hat{N}_{\mathrm{e}}$ based on PwoP is not affected by changes in $\bar{k}$ when null parents are ignored. In the remainder of this study, the term $N_{\mathrm{e}}$ is used to refer to inbreeding effective size.

\section{Reconstructing parental contributions $\left(k_{i}\right)$}

Reconstructing parental genotypes from a sample of progeny is a very challenging exercise that, at present, is only feasible under special circumstances. Fortunately, doing this is not a specific requirement of estimating effective size using PwoP; all that is required is to be able

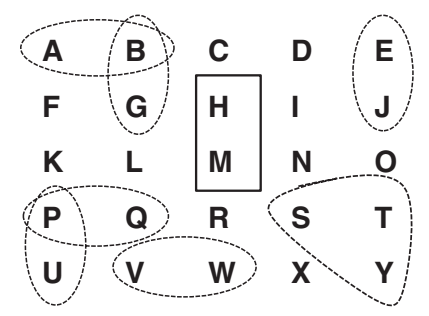

Fig. 1 Sibling relationships in a hypothetical sample of $S=25$ progeny, each denoted by a letter. Unconnected letters (C, D, F ...) represent unrelated individuals; solid rectangle includes two full siblings $(H+M)$; dotted curves include pairs $(V+W)$ or trios $(\mathrm{S}+\mathrm{T}+\mathrm{Y})$ of half-siblings that all share exactly one parent. From these relationships, a vector of $k_{i}$ values can be deduced. Each unrelated individual has two parents with $k_{i}=1$; a pair of full sibs implies two parents, each with $k_{i}=2$; a pair of half-sibs implies one parent with $k_{i}=2$ and two with $k_{i}=1$; and an interlocking pair of half-sib pairs (e.g., $\mathrm{A}+\mathrm{B}+\mathrm{G}$ ) implies 2 parents with $k_{i}=2$ and two with $k_{i}=1$. A trio of half-sibs sharing one parent can be produced in two different ways, both of which result in $\Sigma\left(k_{i}\right)=6$ and $\sum\left(k_{i}^{2}\right)=12$ (see text). In total, 40 different parents are required to produce the depicted relationships (one with $k_{i}=3$, eight with $k_{i}=2$, and 31 with $k_{i}=1$, which yields $\Sigma\left(k_{i}\right)=2 S=50, \sum\left(k_{i}^{2}\right)=72$, and $N_{\mathrm{e}} I=111$ (from eqn 2$)$ ).

to construct the vector of parental contributions (the $k_{i}$ values), and this can be accomplished (or at least attempted) using information provided by sibship reconstruction programmes that are currently available (Jones et al. 2010). Figure 1 illustrates how information on pairwise relationships (full-sib, half-sib, unrelated) can be used to infer parental contributions. In this example, each progeny in a sample of $S=25$ individuals is represented by a letter $(\mathrm{A}-\mathrm{Y})$. Isolated letters $(\mathrm{C}, \mathrm{D}, \mathrm{F} \ldots)$ represent individuals that are not related to any other sampled progeny; each of these progeny must therefore have two unique parents, each with $k_{i}=1$. Solid rectangles connect full siblings (e.g., $\mathrm{H}+\mathrm{M}$ ), and dotted ovals connect pairs of half-sibs (e.g., $\mathrm{V}+\mathrm{W}$; $\mathrm{E}+\mathrm{J}$ ). A pair of full siblings implies two parents each with $k_{i}=2$, whereas a pair of half-siblings implies one parent with $k_{i}=2$ and two parents with $k_{i}=1$. More complicated, interconnected patterns of relationship are of course possible, but each can be decomposed into subsets that allow one to infer the vector of parental contributions. The only ambiguous situation of which we are aware involves a trio of individuals that are reciprocal half-siblings (e.g., offspring S, T, Y in Fig. 1). This pattern can result from either of two mating patterns: (i) one parent is responsible for all three offspring, and three parents contribute one offspring each $\left(k_{i}=3,1,1,1\right)$ or (ii) three parents are each responsible for two of the three offspring $\left(k_{i}=2,2,2\right)$. However, the latter scenario requires at least one individual to reproduce as both male and female so is only possible for hermaphrodites. Furthermore, both of these scenarios lead to $\Sigma k_{i}=6$ and $\Sigma k_{i}^{2}=12$, so the ambiguity has no effect on 
$N_{\mathrm{e}}$ calculated using PwoP (eqn 2). In total, 40 different parents are required to produce the relationships depicted in Fig. 1 (1 parent with $k_{i}=3,8$ with $k_{i}=2$, and 31 with $\left.k_{i}=1\right)$. These parental contributions produce $\Sigma\left(k_{i}\right)=2 S=50, \sum\left(k_{i}^{2}\right)=72$, and $N_{\mathrm{e}}=111$ (from eqn 2 ).

We have developed a program (coded in PYTHON 2.6.4, and available from the authors on request) that constructs the vector of $k_{i}$ values and calculates $N_{\mathrm{e}}$ using eqn (2), based on an input file that specifies the relatedness category for each pair of progeny in the sample. The program creates a parental generation with $2 S$ parents as empty groups. Target progeny are considered one at a time, and after evaluation, each is assigned to two distinct parents. Parents are excluded as possibilities if (i) their group contains non-siblings or (ii) another parental group would match more siblings. A half-sibling pair occurs together in only one parental group, while full siblings share both parental assignments. After all progeny are considered, the vector of $k_{i}$ values is computed as the vector of parental group sizes.

\section{Precision and bias}

The analyses aforementioned are based on an optimal scenario: all progeny have been sampled, and all pairwise relationships among the progeny have been ascertained without error. Under these circumstances, a unique solution can be found for the terms $\Sigma\left(k_{i}\right)$ and $\sum\left(k_{i}^{2}\right)$, which allows one to calculate $N_{\mathrm{e}}$ using eqn (2). In most practical applications involving parentage analysis, effective size can only be estimated, in which case it is important to consider two major sources of uncertainty associated with PwoP:

1 Uncertainty associated with sampling only a fraction of the total number of progeny produced;

2 Uncertainty associated with imperfectly resolving the relationship categories.

\section{Sampling error}

To focus on the first issue (random errors associated with sampling progeny), we assume that relationship categories are identified correctly and that an unknown number of progeny are produced, but we have only sampled $S$ of them. The true effective size would be revealed if we could sample all progeny. An important question thus becomes, "What is the relationship between an estimate of $N_{\mathrm{e}}$ and true $N_{\mathrm{e}}$, when the estimate is computed from a sample of progeny using PwoP?" Intuitively, two a priori considerations suggest that such an estimate might be subject to bias: (i) the analysis leading to eqn (2) used the parametric formula for variance rather than the finite-sample formula and (ii) the vector of $k_{i}$ values generated by PwoP based on only a sample of progeny will not only fail to include null parents, but it will also fail to include any parents that actually did produce offspring but whose offspring (by chance) did not appear in the sample.

To empirically evaluate these factors, we used a Wright-Fisher model (discrete generations, constant $N$, random mating but no selfing, each parent with an equal opportunity to produce offspring) to simulate production of $S$ progeny whose parents were randomly chosen from $N$ potential parents. As no selfing was allowed, we used a slight modification to eqn (1) that is appropriate for species that have separate sexes or are monoecious but avoid selfing:

$$
N_{\mathrm{e}}=\frac{\bar{k} N-2}{\bar{k}-1+V_{k} / \bar{k}}
$$

(Crow \& Denniston 1988, Equation 2). Making the substitutions above for $\bar{k}$ and $V_{k}$ leads to (inbreeding $N_{\mathrm{e}}$, without selfing)

$$
N_{\mathrm{e}}=\frac{2 S-2}{\frac{\sum\left(k_{i}^{2}\right)}{2 S}-1}
$$

Results of these simulations (Table 1), which can be considered 'optimal PwoP' because they assume the vector of $k_{i}$ values can be assembled without error, provide important information about both bias and precision. First, analysing only a sample of progeny has virtually no systematic effect on $\hat{N}_{\mathrm{e}}$ computed by PwoP: harmonic mean $\hat{N}_{\mathrm{e}}$ was very close to the nominal $N$ regardless of the number of progeny sampled. We also verified through simulations that essentially unbiased estimates are also obtained from PwoP using eqn (6), when sexes are separate and sex ratio is skewed (data not shown, but see Fig. 4). Second, unless $N$ is large and $S$ is small, PwoP estimates have relatively small coefficients of variation (CV). For example, a $\mathrm{CV} \leq 0.35$ can be achieved for $N=50-100$ with $S \geq 25$ and for $N=500-1000$ with $S \geq 100$, and much smaller CVs are possible with larger samples. However, if $N$ is large and $S$ is small, $\hat{N}_{\mathrm{e}}$ from PwoP will have very wide confidence intervals (note very large CVs for $S=25, N=500$ and $S=25-50, N=1000$; Table 1). In these scenarios, the distribution of $\hat{N}_{\mathrm{e}}$ is highly skewed toward large values. This occurs because, as fewer and fewer siblings are identified, most of the $k_{i}$ values are 1 . As a result, $\sum\left(k_{i}^{2}\right)$ approaches $\sum\left(k_{i}\right)$, and the denominator of eqn (2) approaches zero, leading to a large $\hat{N}_{\mathrm{e}}$. In the limit, if all progeny are unrelated, this implies that each parent produces exactly one offspring, and $\hat{N}_{\mathrm{e}}$ using eqns (2) or (6) becomes infinitely large. This makes biological sense, as finding no related individuals is the expected result when sampling progeny from an 
Table 1 Assessment of bias and precision in estimates of $N_{\mathrm{e}}$ based on samples of progeny analysed with PwoP. This analysis assumes sibship reconstruction can be done without error ('Optimal PwoP'). Values shown are the harmonic mean $\hat{N}_{\mathrm{e}}$ (and coefficient of variation of $\hat{N}_{\mathrm{e}}$ ) for 10000 simulated Wright-Fisher populations, where parents of $S$ progeny were randomly chosen from $N$ potential parents. No selfing was allowed, so $\hat{N}_{\mathrm{e}}$ was calculated using eqn (6)

\begin{tabular}{llrll}
\hline & \multicolumn{4}{c}{ Parental population $(N)$} \\
\cline { 2 - 5 } $\begin{array}{l}\text { Sample } \\
\text { size }(S)\end{array}$ & 50 & \multicolumn{1}{c}{100} & 500 & 1000 \\
\hline 25 & $49.3(0.21)$ & $98.7(0.35)$ & $483.7(550)$ & $1026.2(454)$ \\
50 & $49.4(0.1)$ & $100.0(0.14)$ & $494.4(0.44)$ & $1018.1(84)$ \\
100 & $49.7(0.05)$ & $99.7(0.07)$ & $501.6(0.17)$ & $994.2(0.26)$ \\
200 & $49.9(0.02)$ & $99.7(0.03)$ & $498.3(0.08)$ & $996.4(0.11)$ \\
\hline
\end{tabular}

infinite number of parents. In the simulations, these infinite $\hat{N}_{\mathrm{e}}$ values were recorded as $10^{6}$ to simplify calculations of $\mathrm{CV}\left(\hat{N}_{\mathrm{e}}\right)$. Therefore, the exact $\mathrm{CV}$ values for these scenarios should be interpreted with caution; the salient point is that, if $S$ is too small compared to $N_{\mathrm{e}}$, the distribution of $\hat{N}_{\mathrm{e}}$ can be highly skewed toward large (and potentially infinite) values. A similar result is found for other estimators of contemporary $N_{\mathrm{e}}$ (discussed by Waples \& Do 2010).

It is useful to consider the probability of obtaining an estimate of $\hat{N}_{\mathrm{e}}=\infty$ based on PwoP. If $\hat{N}_{\mathrm{e}}=\infty$, the $S$ progeny must have $2 S$ unique parents. If we assume a Wright-Fisher model with random selfing, this outcome requires that $2 S$ random draws, with replacement, have been made from $N$ potential parents without having any parent drawn more than once. The probability of this occurring is $N / N \times(N-1) / N \times(N-2) / N \ldots \times(N-2 S+1) /$ $N$, which can be written as

$$
\operatorname{Prob}(\text { no sibs })=\frac{N !}{(N-2 S) ! N^{2 S}}
$$

If the population is randomly mating but not ideal (i.e., some individuals have a higher probability of being a parent than others), eqn (7) should still be valid if $N_{\mathrm{e}}$ is substituted for $N$.

Table 2 shows results of applying eqn 7 to various combinations of $S$ and $N_{\mathrm{e}}$. It is apparent that in many circumstances, only modest sample sizes are required before the probability of an infinite estimate becomes very low. For example, with true $N_{\mathrm{e}}=100$, an infinite $\hat{N}_{\mathrm{e}}$ is very unlikely if 15 or more progeny have been sampled, and with $N_{\mathrm{e}}=200$, an infinite $\hat{N}_{\mathrm{e}}$ is even more unlikely if at least 25 progeny have been sampled. However, with $N_{\mathrm{e}}=1000$, finding no relatives will be quite common in samples of 25 progeny or less, and the probability of an infinite $\hat{N}_{\mathrm{e}}$ does not drop below $1 \%$ until sample size is almost 50 progeny. These results explain the high $\mathrm{CV}$
Table 2 Probability (from eqn 7) of finding no related individuals (and hence an infinite estimate of $N_{\mathrm{e}}$ ) in a random sample of $S$ progeny produced by $N_{\mathrm{e}}=N$ effective parents, assuming random selfing. Last column shows the maximum finite $\hat{N}_{\mathrm{e}}$ from eqn (8) for each sample size

\begin{tabular}{lccc}
\hline$N$ & $S$ & $\begin{array}{c}\text { Probability } \\
\text { (no sibs) }\end{array}$ & $\begin{array}{c}\text { Maximum } \\
\text { finite } \hat{N}_{\mathrm{e}}\end{array}$ \\
\hline 50 & 5 & 0.382 & 45 \\
50 & 10 & 0.012 & 190 \\
50 & 15 & $<10^{-4}$ & 435 \\
100 & 10 & 0.130 & 190 \\
100 & 15 & 0.008 & 435 \\
100 & 20 & $<10^{-3}$ & 780 \\
200 & 10 & 0.374 & 190 \\
200 & 15 & 0.101 & 435 \\
200 & 20 & 0.015 & 780 \\
200 & 25 & 0.001 & 1225 \\
500 & 15 & 0.412 & 435 \\
500 & 25 & 0.079 & 1225 \\
500 & 30 & 0.025 & 1770 \\
500 & 40 & 0.001 & 3160 \\
500 & 50 & $<10^{-4}$ & 4950 \\
1000 & 15 & 0.644 & 435 \\
1000 & 25 & 0.288 & 1225 \\
1000 & 40 & 0.039 & 3160 \\
1000 & 50 & 0.006 & 4950 \\
1000 & 60 & $<10^{-3}$ & 7140 \\
\hline & & &
\end{tabular}

values shown in Table 1 for some simulation scenarios. With only 25 progeny sampled, we expect the fraction of $\hat{N}_{\mathrm{e}}=\infty$ estimates to be $8 \%$ for $N_{\mathrm{e}}=500$ and $29 \%$ for $N_{\mathrm{e}}=$ 1000. The high CV for $S=50, N_{\mathrm{e}}=1000$ was also influenced by $\sim 0.6 \%$ infinite estimates (recorded as $10^{6}$ ).

It is also easy to calculate the upper bound for finite $\hat{N}_{\mathrm{e}}$, which occur if exactly one related pair of progeny is identified. With a single pair of half-sibs, the vector of $k_{i}$ values includes $2 S-2$ parents with $k_{i}=1$ and one parent with $k_{i}=2$. This leads to $\sum\left(k_{i}\right)=2 S$ and $\sum\left(k_{i}^{2}\right)=2 S+2$, and eqn (2) becomes

$$
\text { Maximum finite } \hat{N}_{\mathrm{e}}=\frac{2 S-1}{\frac{2 S+2}{2 S}-1}=2 S^{2}-S \quad \text { (eqn 8) }
$$

Table 2 shows results of applying eqn (8) to the combinations of $S$ and $N$ values discussed earlier. One noteworthy result is that if sample size is very small $(S=5-15$, depending on true $N_{\mathrm{e}}$ ), the maximum finite $\hat{N}_{\mathrm{e}}$ can be less than the true $N_{\mathrm{e}}$. In that case, $\hat{N}_{\mathrm{e}}$ is unreliable even though the harmonic mean is essentially unbiased, because the distribution has a mix of finite estimates less than the true $N_{\mathrm{e}}$ balanced by a fraction of infinite estimates. If true $N_{\mathrm{e}}$ is relatively large, then larger samples of progeny are needed to produce a more balanced distribution of potential $\hat{N}_{\mathrm{e}}$ values. For example, for $S=25$, the largest possible finite estimate of $N_{\mathrm{e}}$ is 1225 (Table 2), 
which is more than twice as large as 500 but only marginally higher than 1000 . If true $N_{\mathrm{e}}$ is 1000 , therefore, sampling only 25 individuals would make it impossible for the distribution of $\hat{N}_{\mathrm{e}}$ to be clustered around the true value.

Finally, we used simulated data to compare the distribution of $\hat{N}_{\mathrm{e}}$ values from optimal PwoP with those obtained using a single-sample method for estimating $N_{\mathrm{e}}$ based on linkage disequilibrium (LD). To generate genetic data for the LD method, we used EasyPop (Balloux 2001) to simulate a Wright-Fisher model with equal numbers of each sex. We generated 'microsatellite' data for independent gene loci using a mutation rate of $5 \times 10^{-4}$ and a $\mathrm{K}$-allele model with a maximum of 10 allelic states and initiated the simulation using the Maximal diversity option. After running the simulation for enough generations $(\geq 25)$ to produce average heterozygosities in the range $(\sim 0.8)$ typically found in many natural populations, we sampled genotypes from $S$ progeny and used these to estimate $N_{\mathrm{e}}$ using the program LDNE (Waples \& Do 2008). LDNE implements a bias correction (Waples 2006) to the standard Hill (1981) method; we report results after excluding alleles with frequency $<0.02$ (as suggested by Waples \& Do 2010). PwoP estimates were generated as in Table 1 using the same $S$ and $N$ values, but using eqn (6) because the genetic data were generated with separate sexes. We considered two scenarios, with true $N_{\mathrm{e}}=100$ or 1000 . The sample size $(S=50)$ and number of loci (20) used are comparable to those used in many contemporary studies of effective size in natural populations.

As shown in Fig. 2, the distribution of $\hat{N}_{\mathrm{e}}$ from PwoP was much narrower than for the LD method. This was especially true for $N_{\mathrm{e}}=1000$, in which case PwoP reduced by a factor of nearly four the fraction of estimates that were more than twice the true $N_{\mathrm{e}}$ (from nearly $40 \%$ for the LD method to just over $10 \%$ for PwoP; Fig. 2A). Even with true $N_{\mathrm{e}}=100$, for which LD estimates are relatively robust, the distribution of PwoP estimates was less biased and substantially tighter (harmonic mean $\hat{N}_{\mathrm{e}}=99.7$ (range 64-171) for PwoP compared to 113.9 (63-376) for LD; Fig. 2B).

\section{Errors in sibship reconstruction}

Evaluations of precision and bias in the previous section represent best-case ('optimal') scenarios for PwoP, because they assumed that sibships were accurately determined. Strictly speaking, this assumption is not required for the aforementioned results to be accurate, because the validity of eqn (2) depends only on the term $\sum\left(k_{i}^{2}\right)$ and not the exact pattern of relationship or the entire vector of $k_{i}$ values. It would be possible, for example, for some errors to occur in sibship reconstruc-

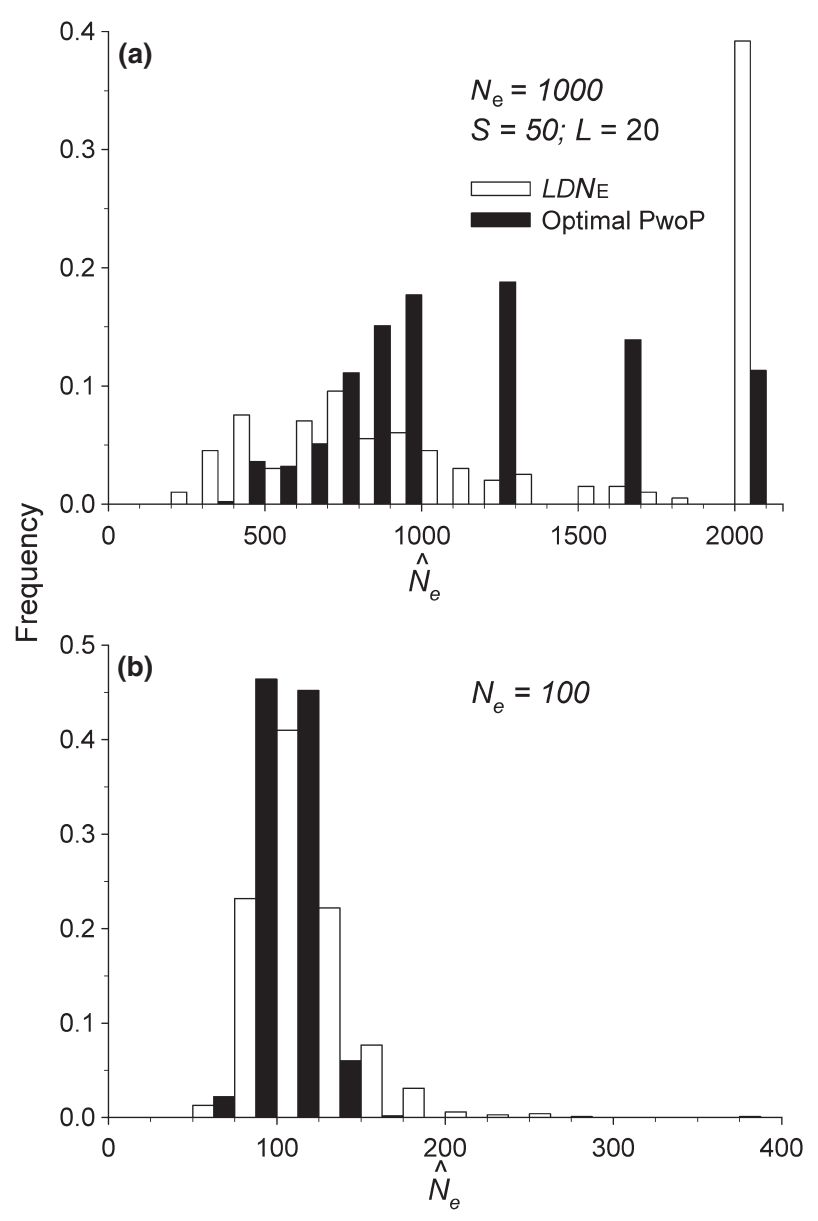

Fig. 2 Distribution of $\hat{N}_{\mathrm{e}}$ for simulated Wright-Fisher populations with true $N_{\mathrm{e}}=1000$ (a) or 100 (b) using two methods. LDNe used $L=20$ 'microsat' loci, and optimal PwoP assumed the vector of $k_{i}$ values was created without error. Both used samples of $S=50$ individuals. In (a), the last bin on the right includes all estimates $>2000$.

tion that do not affect $\sum\left(k_{i}^{2}\right)$ and hence do not affect $\hat{N}_{\mathrm{e}}$ from PwoP. Nevertheless, sibship reconstruction without parental genotypes as a guide is such a challenging task that uncertainty in this step will have a large influence on practical utility of PwoP.

A rigorous evaluation of this topic should consider two related issues: (i) limited power to resolve relationship categories given finite samples of progeny and gene loci and (ii) effects of genotypic errors. These two factors interact to affect bias and precision of sibship reconstructions, and they also involve tradeoffs that differ qualitatively depending on the markers used. For example, each microsatellite locus typically has many more alleles and hence more power for sibship reconstruction or parentage analysis than does a single single-nucleotide polymorphism (SNP) marker, but microsatellites also typically have higher genotyping error rates. Unless the 
per-locus genotyping error rate is very low, as the number of loci increases the chances than any given individual will have at least one mis-scored genotype can become quite high, which can bias results even as theoretical power increases (e.g., see Anderson \& Garza 2006).

We will not attempt a comprehensive assessment of this complex topic here. However, we want to provide some indication of the performance of PwoP that can be achieved with currently available software. Jones et al. (2010) listed seven freely available programs that attempt sibship reconstruction, but most of these consider only full sibs or partial subsets of half-sibs. Only two of the programs [Colony (Jones \& Wang 2010) and ML-Relate (Kalinowski et al. 2006)] attempt the generalized sibship reconstruction envisioned by PwoP. Of these, Colony in theory should provide more robust results, as it jointly considers the likelihood of larger patterns of relationship, whereas ML-ReLATE independently determines the relationship of each pair of progeny. However, ColONY is computationally intensive and not easily adapted for simulation studies, while ML-RELATE is simple and quick and can read simulated genetic data in standard formats. Accordingly, we conducted a few exploratory runs in which ML-ReLATE was challenged with simulated genotypic data (using EasyPop as described above), and the resulting determinations of pairwise relationships were used to calculate $\hat{N}_{\mathrm{e}}$ using PwoP. ML-Relate uses maximum likelihood to independently find the most likely relationship category for each progeny pair (parent-offspring, full sibling, half-sibling, unrelated). Because we simulated discrete-generation data, our samples of progeny had no parent-offspring dyads, so any parent-offspring determinations by ML-RELATE were scored according to the next most likely relationship category (which most frequently was half-sib).

Figure 3 shows results of analyses comparable to those in Fig. 2B (20 'microsat' loci, $S=50, N_{\mathrm{e}}=50,100$, 200). Under these conditions, precision using PwoP and ML-RELATE was actually quite high, but substantial biases were apparent. For example, with true $N_{\mathrm{e}}=100$, uncertainty in sibship reconstruction led to a sharp downward bias in ML-RelATE estimates (harmonic mean $\hat{N}_{e(\mathrm{ML}-\mathrm{R})}=78.5$; all estimates fell in the range 65-98). The downward bias was more pronounced for true $N_{\mathrm{e}}=200$ (harmonic mean $\hat{N}_{e(\mathrm{ML}-\mathrm{R})}=101.8$; range $=78-123$ ), but for $N_{\mathrm{e}}=50$, the bias was modest and positive (harmonic mean $\hat{N}_{e(\mathrm{ML}-\mathrm{R})}=56.8$; range $\left.=48-70\right)$. These results suggest an interaction between sample size and effective size with respect to bias of $\hat{N}_{\mathrm{e}}$ using ML-ReLATE.

A likely explanation for this downward bias is overestimation of the number of pairs of progeny that are related, which would inflate the estimate of $\sum\left(k_{i}^{2}\right)$ and lead to an underestimate of $N_{\mathrm{e}}$. Note that this can occur

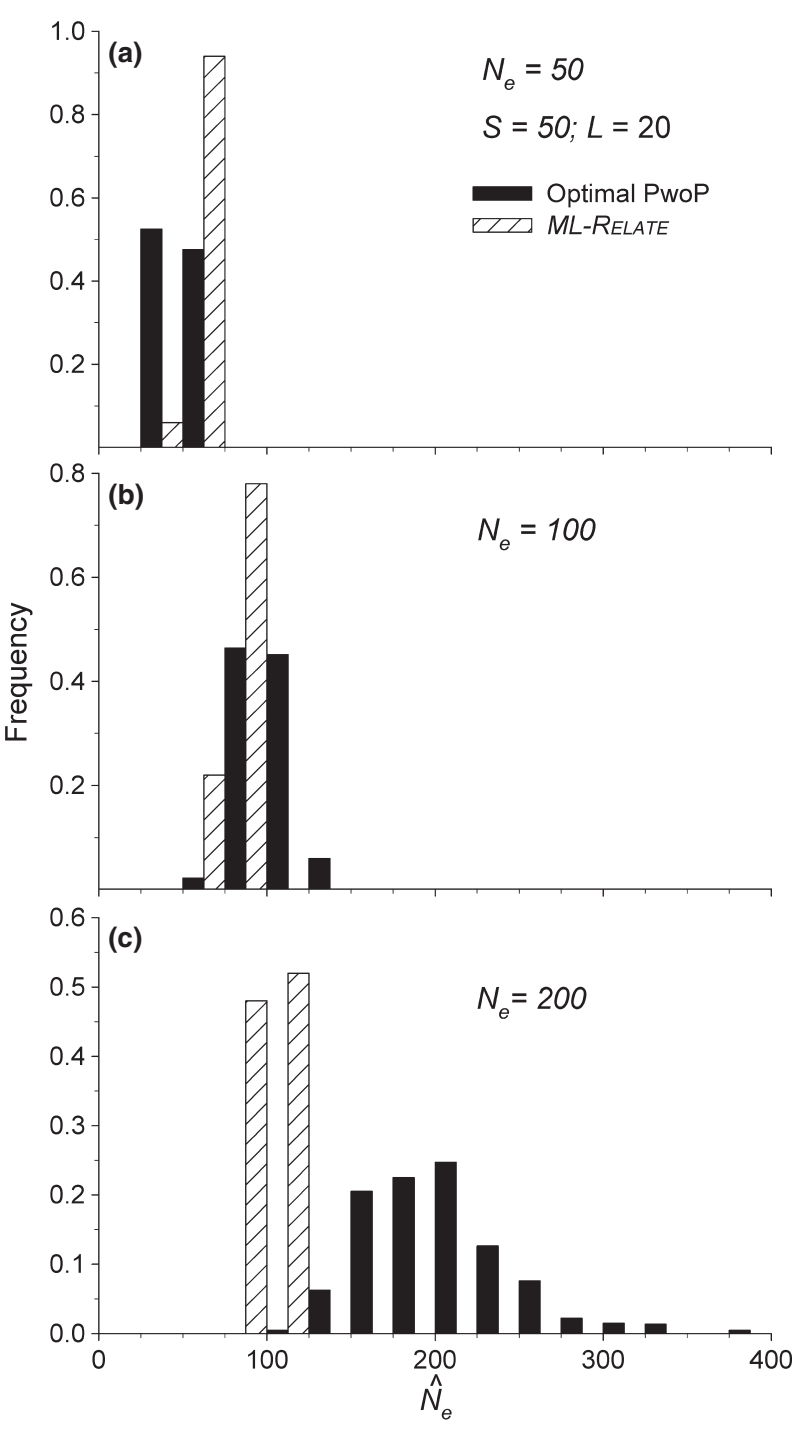

Fig. 3 Distribution of $\hat{N}_{\mathrm{e}}$ for PwoP estimates when true $N_{\mathrm{e}}$ was 50 (a), 100 (b) or 200 ideal individuals (c). Each panel compares estimates based on (i) simulated demographic data under optimal conditions (assuming perfect sibship reconstruction, as in Fig. 2; black bars) and (ii) simulated genotypic data (using 20 'microsat loci', as in Fig. 2 for LDNE) analysed using the program ML-ReLATE (hatched bars).

even if the probability of making a Type I error (wrongly inferring too high a relationship category) is lower than the probability of a Type II error (wrongly inferring too low a relationship category) - as reported previously for ML-Relate (Kozfkay et al. 2008) and for another relationship estimation program (Thomas \& Hill 2002). Unless $N$ is very small, truly unrelated pairs will comprise most of the progeny, so even a low Type I error rate can lead to net estimates of more siblings than actually exist. We believe that this factor was responsible for the downward bias in $\hat{N}_{\mathrm{e}}$ for $N=100$ and 200. Although sibships were 
also generally overestimated for $N=50$, another factor appears to have been relatively more important in that case: impossible combinations of relationships that can occur as a result of relying on strictly pairwise analyses (e.g., A and B are determined to be full sibs, as are A and $\mathrm{C}$, but $\mathrm{B}$ and $\mathrm{C}$ are not). The simulations with $N=50$ had a relatively high frequency of these impossible combinations, which neither ML-RELATE nor our simple algorithm were designed to try to resolve. In our simple algorithm, these incompatibilities hinder the formation of large sibling groups under a single parent; this reduces the fraction of large $k_{i}$ values [and reduces $\sum\left(k_{i}^{2}\right)$ ] and tends to inflate the estimate of $N_{\mathrm{e}}$.

To evaluate the effects of marker type, we repeated the aforementioned analyses with true $N_{\mathrm{e}}=100$ and either 100 or 200 diallelic ('SNP') loci instead of 20 'microsat' loci. One hundred SNP loci produced results comparable to those for the 'microsat' data: precise but downwardly biased estimates using ML-Relate (harmonic mean $\hat{N}_{\mathrm{e}}=74.8$; range 62-92). However, use of 200 SNPs led to precise estimates with relatively little downward bias (harmonic mean $\hat{N}_{\mathrm{e}}=93.4$; range $78-$ 123) (Fig. 4A).

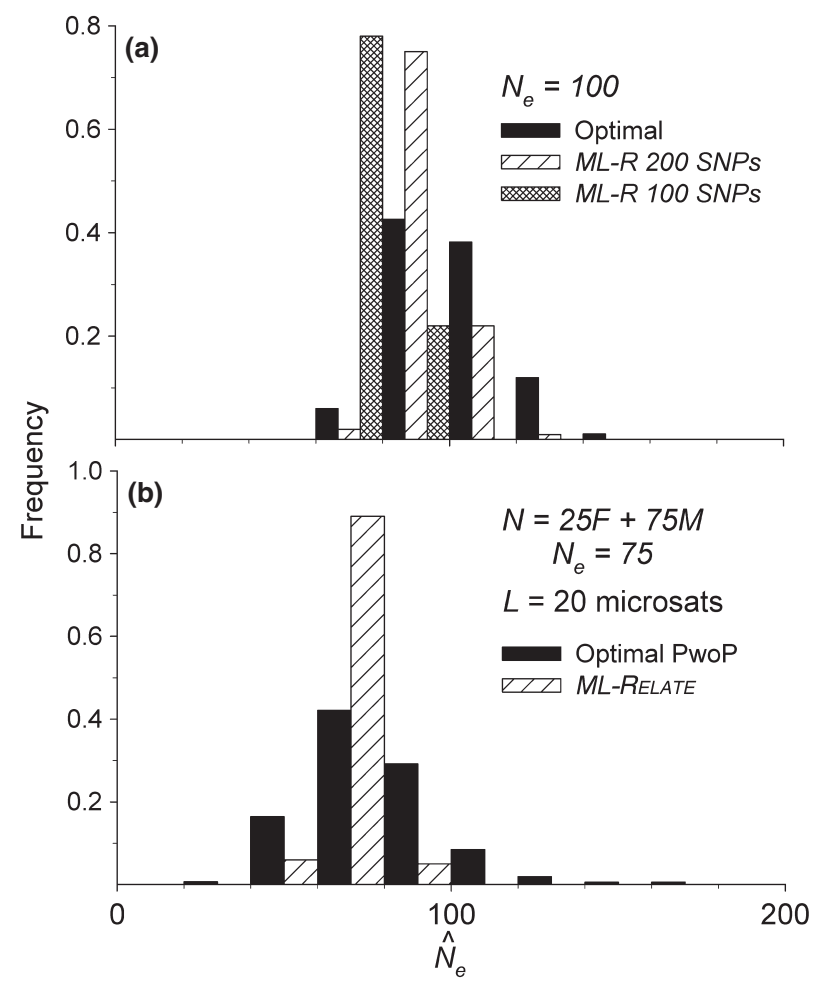

Fig. 4 (a) As in Figure 3, but using either 100 or 200 diallelic 'single-nucleotide polymorphism' loci for the ML-ReLATE estimates. True $N_{\mathrm{e}}$ was 100. (b) Distribution of $\hat{N}_{\mathrm{e}}$ for 'optimal' PwoP estimates when sex ratio was skewed (25 females +75 males; true $N_{\mathrm{e}}=75$ ). ML-Relate estimates used 20 'microsat' loci. In both panels, sample size was $S=50$.
Simulation results presented so far have used ideal Wright-Fisher populations with equal sex ratio. Equations 5 and 6 account for skewed sex ratio, as overall $V_{k}$ increases if the numbers of each sex are not equal, and Fig. 4B shows that 'optimal' PwoP estimates accurately reflect the lowered $N_{\mathrm{e}}$ from a 3:1 sex ratio (harmonic mean $\hat{N}_{\mathrm{e}}=75.7$; range 40-150). Skewed sex ratio also did not adversely affect estimates based on the program MLRelATE: harmonic mean $\hat{N}_{\mathrm{e}}$ was only slightly lower than expected (68.9), and the range of estimates (53-82) was actually tighter than under optimal PwoP (Fig. 4B).

\section{Discussion}

The new formulas (eqns 2 and 6) show that inbreeding effective size can be expressed in terms of a single unknown parameter $\left(\sum\left(k_{i}^{2}\right)\right)$, independent of the total number of parents, $N$ [of course, it is necessary to know or be able to estimate $N$ if one is interested in the $N_{\mathrm{e}} / N$ ratio]. Except for the special case with $\bar{k}=2$, this property is not shared by variance effective size (which depends on the number of progeny sampled), and this emphasizes the point that inbreeding $N_{\mathrm{e}}$ is the more useful measure of effective size for parentage analysis. The advantage of the new formulation is that it allows unbiased calculation of $N_{\mathrm{e}}$ in parentage analysis under a wide range of circumstances. Although the analyses considered here assumed that no parents can be genotyped, the method can also be applied when some but not all potential parents can be identified and sampled-a situation that occurs quite often in studies of natural populations (e.g., Emery et al. 2001; Araki et al. 2007).

Although we have not attempted a rigorous performance evaluation of PwoP, results of the analysis of simulated data establish two major points:

1 PwoP can provide unbiased and precise estimates of $N_{\mathrm{e}}$ from random samples of progeny, provided the vector of $k_{i}$ values can be constructed accurately.

2 Accomplishing the latter will be challenging, and substantial biases can occur if systematic errors occur in reconstructing sibling relationships.

Fortunately, to estimate $N_{\mathrm{e}}$ using PwoP it is not necessary to reconstruct parental genotypes, which is exceedingly challenging unless at least some parents can be genotyped. The analyses described earlier depend only on reconstruction of sibling relationships, which can be estimated using currently available software. It should be possible to improve considerably on the biases indicated in Fig. 3 by adopting more sophisticated methods that jointly consider relationships among groups of related individuals. Performance with 200 'SNP' loci was encouraging (Fig. 4A); however, results obtained by Santure 
et al. (2010) and others caution that use of large numbers of markers will not necessarily achieve the desired level of precision or accuracy. Use of fractional or probabilistic relationship assignments might also be an effective strategy for reducing bias and increasing precision, as could inclusion of other types of information, such as individual phenotypes (Walling et al. 2010).

Although the approach outlined here used sibship reconstructions to infer the vector of $k_{i}$ values, that also is not necessarily required, as the key parameter to estimate is $\sum\left(k_{i}^{2}\right)$ and estimating the full vector of parental contributions is only an intermediate step in the process. This suggests that a more profitable approach might be to develop a method to specifically estimate $\sum\left(k_{i}^{2}\right)$, either directly or jointly with estimating the sibling relationships, as in full probability parentage analysis (Jones et al. 2010; Serbezov et al. 2010).

PwoP has some obvious parallels to Wang's (2009) sibship method for estimating $N_{\mathrm{e}}$. Both use information from sibship reconstructions to estimate effective size; the difference is that Wang's method uses the sibship results to calculate the probability of different relationship categories under different hypotheses for $N_{\mathrm{e}}$, while PwoP uses the sibship results to calculate the parental contributions and hence $N_{\mathrm{e}}$ directly using a demographic formula.

Future research might also focus on comparison of the performance of PwoP with other single-sample estimators besides LDNE (Pudovkin et al. 1996; Nomura 2008; Tallmon et al. 2008; Wang 2009) under a variety of realistic conditions. As discussed by Waples \& Do (2010), calculating a combined estimate of effective size based on results of multiple methods can substantially increase precision (and potentially reduce bias), especially if the methods provide independent information about effective size. For example, Waples (1991) reported that $\hat{N}_{\mathrm{e}}$ from the temporal and LD method are essentially uncorrelated, but comparable evaluations have not been performed for the different single-sample estimators.

Unlike some other genetic methods for estimating $N_{\mathrm{e}}$, PwoP does not depend on the assumption of selective neutrality, as it relies on genetic data only to reconstruct parental contributions, nor does it depend on assumptions about the mating system. Like other estimators of contemporary $N_{\mathrm{e}}$ (and unlike estimators of long-term $N_{\mathrm{e}}$ ), PwoP does not require an estimate of mutation rate, and mutation poses a problem only insofar as it might affect sibship reconstruction. Whether immigration (or other factors that might cause individuals from multiple populations to appear in the sample) represents a problem depends on the objectives and the quantity one is trying to estimate. Unlike some other estimators, PwoP does not depend on theoretical expectations for genetic processes in closed populations. If individuals from more than one population appear in the sample, the immi- grants presumably would be determined to be unrelated to local individuals, which would tend to reduce $\sum\left(k_{i}^{2}\right)$ and increase $\hat{N}_{\mathrm{e}}$. From one point of view, this would accurately reflect the reality that the sample is produced by more parents than occur in just the local population. On the other hand, this could be misleading if the goal is to estimate just the local $N_{\mathrm{e}}$. In the latter case, it might be possible to use genetic assignment methods to exclude immigrants and focus only on locally produced progeny (as suggested by Wang 2009).

Although we did not evaluate this directly, PwoP presumably shares with most or all other estimators of contemporary $N_{\mathrm{e}}$ a sensitivity to sampling from agestructured populations. The standard formulas for effective size that PwoP is based on (eqns 1 and 5) assume discrete generations. If the sample is from a single cohort in an age-structured population, the estimate should be directly interpretable in terms of $N_{\mathrm{b}}$, the effective number of breeders in one year or one breeding season. However, if mixed-age samples are taken from iteroparous species with overlapping generations, the resulting estimate can be difficult to interpret in terms of effective size for a generation as a whole $\left(N_{\mathrm{e}}\right.$; see Waples \& Yokota 2007). More research is needed to better elucidate the relationship between $N_{\mathrm{b}}$ and $N_{\mathrm{e}}$ in age-structured species.

\section{Acknowledgements}

We are indebted to Mark Bravington for sharing unpublished data and to him, James Crow, Peter Smouse, and Ian Wilson for stimulating discussions. Three anonymous reviewers provided useful comments on an earlier draft. This work arose from a project funded by the Gordon and Betty Moore Foundation via the University of California, Santa Barbara and was inspired by code written by Eli Meir from SimBiotic Software.

\section{Conflict of interest}

The authors have no conflict of interest to declare and note that the sponsors of the issue had no role in the study design, data collection and analysis, decision to publish, or preparation of the manuscript.

\section{References}

Anderson EC, Garza JC (2006) The power of single-nucleotide polymorphisms for large-scale parentage inference. Genetics, 172, 2567-2582.

Araki HA, Waples RS, Ardren WR, Cooper B, Blouin MS (2007) Effective population size of steelhead trout: influence of variance in reproductive success, hatchery programs, and genetic compensation between life-history forms. Molecular Ecology, 16, 953-966.

Balloux F (2001) EASYPOP (version 1.7): a computer program for population genetics simulations. Journal of Heredity, 92, 301-302.

Caballero A (1994) Developments in the prediction of effective population size. Heredity, 73, 657-679.

Crow JF (1954) Breeding structure of populations. II. Effective breeding number. In: Statistics and Mathematics in Biology (eds Kempthorne O, 
Bancroft TA, Gowen JW, Lush JL), pp. 543-556. Iowa State College Press, Ames, IA.

Crow JF, Denniston C (1988) Inbreeding and variance effective population numbers. Evolution, 42, 482-495.

Crow JF, Morton NE (1955) Measurement of gene frequency drift in small populations. Evolution, 9, 202-214.

DeWoody JA, Walker D, Avise JC (2000) Genetic parentage in large halfsib clutches: theoretical estimates and empirical appraisals. Genetics, 154, 1907-1912.

Emery AM, Wilson IJ, Craig S, Boyle PR, Noble LR (2001) Assignment of paternity groups without access to parental genotypes: multiple mating and developmental plasticity in squid. Molecular Ecology, 10, 12651278.

Eriksson A, Mehlig B, Panova M, Andre C, Johannesson K (2010) Multiple paternity: determining the minimum number of sires of a large brood. Molecular Ecology Resources, 10, 282-291.

Hill WG (1981) Estimation of effective population size from data on linkage disequilibrium. Genetical Research, 38, 209-216.

Jones AG (2005) GERUD2.0: a computer program for the reconstruction of parental genotypes from progeny arrays with known or unknown parents. Molecular Ecology Notes, 5, 708-711.

Jones AG, Avise JC (1997) Polygynandry in the dusky pipefish Syngnathus floridae revealed by microsatellite DNA markers. Evolution, 51, 16111622.

Jones AG, Small CM, Paczolt KA, Ratterma NL (2010) A practical guide to methods of parentage analysis. Molecular Ecology Resources, 10, 6-30.

Jones OR, Wang J (2010) CoLONY: a program for parentage and sibship inference from multilocus genotypic data. Molecular Ecology Resources, 10, 551-555.

Kalinowski ST, Wagner AP, Taper ML (2006) ML-ReLATE: a computer program for maximum likelihood estimation of relatedness and relationship. Molecular Ecology Notes, 6, 576-579.

Kozfkay CC, Campbell MR, Heindel JA et al. (2008) A genetic evaluation of relatedness for broodstock management of captive, endangered Snake River sockeye salmon, Oncorhynchus nerka. Conservation Genetics, 9, 1421-1430.

Myers EM, Zamudio KR (2004) Multiple paternity in an aggregate breeding amphibian: the effect of reproductive skew on estimates of male reproductive success. Molecular Ecology, 13, 1951-1963.

Nomura T (2008) Estimation of effective number of breeders from molecular coancestry of single cohort sample. Evolutionary Applications, 1, 462-474.

Pudovkin AI, Zaykin DV, Hedgecock D (1996) On the potential for estimating the effective number of breeders from heterozygote-excess in progeny. Genetics, 144, 383-387.
Santure AW, Stapley J, Ball AD, Birkhead TR, Burke T, Slate J (2010) On the use of large marker panels to estimate inbreeding and relatedness: empirical and simulation studies of a pedigreed zebra finch population typed at 771 SNPs. Molecular Ecology, 19, 1439-1451.

Serbezov D, Bernatchez L, Olsen EM, Vøllestad LA (2010) Mating patterns and determinants of individual reproductive success in brown trout (Salmo trutta) revealed by parentage analysis of an entire stream living population. Molecular Ecology, 19, 3193-3205.

Tallmon DA, Koyuk A, Luikart G, Beaumont MA (2008) ONESAMP: a program to estimate effective population size using approximate Bayesian computation. Molecular Ecology Resources, 8, 299-301.

Tatarenkov A, Healy CIM, Grether GF, Avise JC (2008) Pronounced reproductive skew in a natural population of green swordtails, Xiphophorus helleri. Molecular Ecology, 20, 4522-4534.

Thomas SC, Hill WG (2002) Sibship reconstruction in hierarchical population structures using Markov chain Monte Carlo techniques. Genetical Research, 79, 227-234.

Walling CA, Pemberton JM, Hadfield JD, Kruuk LEB (2010) Comparing parentage inference software: reanalysis of a red deer pedigree. Molecular Ecology, 19, 1914-1928.

Wang JL (2004) Sibship reconstruction from genetic data with typing errors. Genetics, 166, 1963-1979.

Wang J (2009) A new method for estimating effective population size from a single sample of multilocus genotypes. Molecular Ecology, 18, 2148-2164.

Waples RS (1991) Genetic methods for estimating the effective size of cetacean populations. Report of the International Whaling Commission, (special issue 13), 279-300.

Waples RS (2002) Evaluating the effect of stage-specific survivorship on the $\mathrm{N}_{e} / \mathrm{N}$ ratio. Molecular Ecology, 11, 1029-1037.

Waples RS (2006) A bias correction for estimates of effective population size based on linkage disequilibrium at unlinked gene loci. Conservation Genetics, 7, 167-184.

Waples RS, Do C (2008) LDNE: a program for estimating effective population size from data on linkage disequilibrium. Molecular Ecology Resources, 8, 753-756.

Waples RS, Do C (2010) Linkage disequilibrium estimates of contemporary $N_{e}$ using highly variable genetic markers: a largely untapped resource for applied conservation and evolution. Evolutionary Applications, 3, 244-262.

Waples RS, Yokota M (2007) Temporal estimates of effective population size in species with overlapping generations. Genetics, 175, 219-233. 